\title{
Commuting Involution Graphs for 3-Dimensional Unitary Groups
}

\author{
Alistaire Everett \\ School of Mathematics \\ The University of Manchester \\ Oxford Road, Manchester, M13 9PL, UK \\ a.everett@maths.manchester.ac.uk
}

Submitted: Feb 7, 2011; Accepted: Apr 28, 2011; Published: May 8, 2011

Mathematics Subject Classification: 05C12, 20E99

\begin{abstract}
For a group $G$ and $X$ a subset of $G$ the commuting graph of $G$ on $X$, denoted by $\mathcal{C}(G, X)$, is the graph whose vertex set is $X$ with $x, y \in X$ joined by an edge if $x \neq y$ and $x$ and $y$ commute. If the elements in $X$ are involutions, then $\mathcal{C}(G, X)$ is called a commuting involution graph. This paper studies $\mathcal{C}(G, X)$ when $G$ is a 3-dimensional projective special unitary group and $X$ a $G$-conjugacy class of involutions, determining the diameters and structure of the discs of these graphs.
\end{abstract}

\section{Introduction}

For a group $G$ and a subset $X$ of $G$, we define the commuting graph, denoted $\mathcal{C}(G, X)$, to be the graph whose vertex set is $X$ with two distinct vertices $x, y \in X$ joined by an edge if and only if $x y=y x$. Commuting graphs first came to prominence in the groundbreaking paper of Brauer and Fowler [6], famous for containing a proof that only finitely many finite simple groups can contain a given involution centralizer. The commuting graphs employed in this paper had $X=G \backslash\{1\}$ - such graphs have played a vital role in recent results relating to the Margulis-Platanov conjecture (see [11]). When $X$ is a conjugacy class of involutions, we call $\mathcal{C}(G, X)$ a commuting involution graph. This special case demonstrated its importance in the (mostly unpublished) work of Fischer [9], which led to the construction of three new sporadic simple groups. Aschbacher [1] also showed a necessary condition on a commuting involution graph for the presence of a strongly embedded subgroup in $G$. The detailed study of commuting involution graphs came to the fore in 2003 with the work of Bates, Bundy, Hart (nèe Perkins) and Rowley, which explored commuting involution graphs for $G$ a symmetric group, or more generally a finite Coxeter group, a special linear group, or a sporadic simple group ([2], [3], [4], [5]). 
Recently some of the remaining sporadic simple groups were addressed in Taylor [12] and Wright [14]. When $G$ is a 4-dimensional projective symplectic group, the structure of $\mathcal{C}(G, X)$ was determined in [8].

We continue the study of $\mathcal{C}(G, X)$ when $G$ is a finite simple group of Lie type of rank 1 and $X$ is a $G$-conjugacy class of involutions. The case when $G$ is a 2-dimensional projective special linear group was addressed in [4]. The well-known structures of $U_{3}\left(2^{a}\right)$ and $\mathrm{Sz}\left(2^{2 \mathrm{a}+1}\right)$ where $a \in \mathbb{N}$ quickly reveal the commuting involution graphs are disconnected with the connected components are cliques. So the 3-dimensional projective unitary groups of odd characteristic and the Ree groups of characteristic 3 remain to be studied. This paper concentrates on the 3-dimensional unitary groups and from now on, we set $q=p^{a}$ for $p$ an odd prime and $a \in \mathbb{N}$. Let $H=S U_{3}(q)$ and let $X$ be the $H$-conjugacy class of involutions. For $t \in X$ we define the $i^{\text {th }}$ disc to be $\Delta_{i}(t)=\{x \in X \mid d(t, x)=i\}$ where $d$ is the standard distance metric on $\mathcal{C}(H, X)$. Our main theorem is as follows.

Theorem 1.1 $\mathcal{C}(H, X)$ is connected of diameter 3, with disc sizes

$$
\begin{aligned}
& \left|\Delta_{1}(t)\right|=q(q-1) ; \\
& \left|\Delta_{2}(t)\right|=q(q-2)\left(q^{2}-1\right) ; \text { and } \\
& \left|\Delta_{3}(t)\right|=(q+1)\left(q^{2}-1\right) .
\end{aligned}
$$

We remark that for $G=H / Z(H) \cong U_{3}(q)$ and $X_{G}=X Z(H) / Z(H)$, the graphs $\mathcal{C}(H, X)$ and $\mathcal{C}\left(G, X_{G}\right)$ are isomorphic. The proof of Theorem 1.1 is constructive, determining the graph structure as one "steps around the graph". With an appropriately chosen $t$, Lemma 2.3 shows that one can identify which disc a given involution $x \in X$ lies in, by inspection of its top-left entry. It is interesting to note that the third disc is a single $C_{H}(t)$-orbit if and only if $q \not \equiv 5(\bmod 6)$, otherwise it splits into three $C_{H}(t)$-orbits. The collapsed adjacency graphs for both cases are given in [7]. Our group theoretic notation is standard, as given in [10].

\section{The Structure of $\mathcal{C}(G, X)$}

This section gives a proof of Theorem 1.1. Let $V$ be the unitary $G F\left(q^{2}\right) H$-module with basis $\left\{e_{i}\right\}$ and define the unitary form on $V$ by $\left(e_{i}, e_{j}\right)=\delta_{i j}$. Hence the Gram matrix of this form is the identity matrix, and $H$ can be explicitly described as

$$
H=\left\{A \in S L_{3}\left(q^{2}\right) \mid \bar{A}^{T} A=I_{3}\right\} \cong S U_{3}(q) .
$$

For $\alpha \in G F\left(q^{2}\right)$ we set $\bar{\alpha}=\alpha^{q}$, and $\overline{\left(a_{i j}\right)}=\left(\overline{a_{i j}}\right)$. For a matrix $g$, define $g_{i j}$ to be its $(i, j)^{\text {th }}$ entry. There is only one class of involutions in $H$, which we denote by $X$, and fix a representative $t=\left(\begin{array}{rrr}1 & 0 & 0 \\ 0 & -1 & 0 \\ 0 & 0 & -1\end{array}\right)$. 
Lemma 2.1 (i) $\left.C_{H}(t)=\left\{\begin{array}{l|l|l}(a d-b c)^{-1} & & \\ \hline & a & b \\ & c & d\end{array}\right) \mid \begin{array}{c}a, b, c, d \in G F\left(q^{2}\right) \\ \bar{a} a+\bar{c} c=\bar{b} b+\bar{d} d=1 \\ a d-b c \neq 0 \\ \bar{a} b+\bar{c} d=\bar{b} a+\bar{d} c=0\end{array}\right\} \cong G U_{2}(q)$.

(ii) $|X|=q^{2}\left(q^{2}-q+1\right)$.

(iii) $\left|\Delta_{1}(t)\right|=q(q-1)$.

(iv) If $x \in \Delta_{1}(t)$, then $\left|\Delta_{1}(t) \cap \Delta_{1}(x)\right|=1$.

Proof Clearly

$$
C_{H}(t)=\left\{\left(\begin{array}{l|l}
\operatorname{det} A^{-1} & \\
\hline & A
\end{array}\right) \mid A \in G U_{2}(q)\right\} \cong G U_{2}(q)
$$

proving (i).

Part (ii) follows from the fact that $|H|=q^{3}\left(q^{3}+1\right)\left(q^{2}-1\right)$ and $\left|G U_{2}(q)\right|=q(q+1)\left(q^{2}-1\right)$. Let $x=\left(\begin{array}{l|l}\operatorname{det} A^{-1} & \\ \hline & A\end{array}\right) \in C_{H}(t) \cap X$. Using a result of Wall [13], there are two classes of involutions in $G U_{2}(q)$, represented by $-I_{2}$ and $\left(\begin{array}{cc}-1 & 0 \\ 0 & 1\end{array}\right)$. If $A=-I_{2}$, then $x=t$. Assume then that $A$ is the latter choice, giving $\Delta_{1}(t)=x^{C_{G}(H)}$. By a routine calculation as in part (i), it is easy to see that

$$
C_{H}(x)=\left\{\left(\begin{array}{l|l}
A & \\
\hline & \operatorname{det} A^{-1}
\end{array}\right) \mid A \in G U_{2}(q)\right\}
$$

and so

$$
C_{H}(\langle t, x\rangle)=\left\{\left(\begin{array}{ccc}
a & 0 & 0 \\
0 & b & 0 \\
0 & 0 & (a b)^{-1}
\end{array}\right) \mid a, b \in G F\left(q^{2}\right), \bar{a} a=\bar{b} b=1\right\}
$$

with $\left|C_{H}(\langle t, x\rangle)\right|=(q+1)^{2}$. Hence $\left|\Delta_{1}(t)\right|=\frac{\left|C_{H}(t)\right|}{\left|C_{H}(\langle t, x\rangle)\right|}=q(q-1)$, proving (iii), while (iv) follows immediately from the structure of $C_{H}(\langle t, x\rangle)$.

$$
\text { Henceforth, we set } x=\left(\begin{array}{rrr}
-1 & 0 & 0 \\
0 & -1 & 0 \\
0 & 0 & 1
\end{array}\right) \in \Delta_{1}(t)
$$

Lemma 2.2 (i) Let $g, h \in \Delta_{2}(t)$. If $g_{11} \neq h_{11}$, then $g$ and $h$ are not $C_{H}(t)$-conjugate.

(ii) $\Delta_{2}(t) \cap \Delta_{1}(x)=\left\{\left(\begin{array}{cc|c}\frac{a}{b} & b & \\ \hline b & -a & \\ \hline & & -1\end{array}\right) \mid \bar{b} b=1-a^{2}, a \in G F(q) \backslash\{ \pm 1\}\right\}$.

(iii) For each $a \in G F(q) \backslash\{ \pm 1\}$, there are $q+1$ elements $g$ of $\Delta_{2}(t) \cap \Delta_{1}(x)$ such that $g_{11}=a$. 
Proof By an analogous method to that in Lemma 2.1(i), it is clear that

$$
\Delta_{1}(x)=\left\{\left(\begin{array}{cc|c}
a & b & \\
c & -a &
\end{array}\right) \mid a, b, c \in G F\left(q^{2}\right), a^{2}+b c=1\right\} .
$$

Let

$$
g=\left(\begin{array}{cc|c}
a & b & \\
c & -a & \\
\hline & & -1
\end{array}\right) \in \Delta_{1}(x),
$$

for $a, b, c \in G F\left(q^{2}\right)$, and $h \in C_{H}(t)$. Now $\left(h^{-1} g h\right)_{11}=h_{11}^{-1} a h_{11}=a$ and so any two $C_{H}(t)$-conjugate elements have the same top-left entry, so proving (i).

If $b=0$ then $a^{2}+b c=a^{2}=1$ and so $a= \pm 1$. But then $\bar{a} a=1$ and thus $\bar{c} c=0$ implying $c=0$. Similarly, if $c=0$ then $b=0$. If $a= \pm 1$, then $1+b c=1$ and so $b c=0$. Hence, either $b=0$ or $c=0$ and therefore both are 0 . However, $a=1$ implies $g=t$, and $a=-1$ implies $g \in \Delta_{1}(t)$. Therefore if $a= \pm 1$, then $g \notin \Delta_{2}(t)$. In particular, if $a \neq \pm 1$ then $g \in \Delta_{2}(t)$, since $d(t, x)=1$ and $[g, x]=1$. Suppose now $a \neq \pm 1$, so $b, c \neq 0$. Then by Lemma 2.1(i), we have $\bar{a} a+\bar{c} c=\bar{a} a+\bar{b} b=1$ and $\bar{a} b=a \bar{c}$. Therefore $\bar{a} a+\bar{c} c=a^{2} \bar{c} b^{-1}+\bar{c} c=1$ and so $a^{2} b^{-1}+c=\overline{c^{-1}}$. It follows that $b \bar{c}^{-1}=a^{2}+b c=1$ and hence $b=\bar{c}$. However, this yields $\bar{a}=a$, implying $a \in G F(q) \backslash\{ \pm 1\}$, proving (ii).

By combining parts (i) and (ii), $\Delta_{1}(x) \cap \Delta_{2}(t)$ is partitioned into $C_{H}(\langle t, x\rangle)$-orbits, with the action of $C_{H}(\langle t, x\rangle)$ leaving the diagonal entries unchanged. Since $a \neq \pm 1, \bar{b} b \neq 0$ and $\bar{b} b-\left(1+a^{2}\right)=0$. Since there are $q+1$ solutions in $G F\left(q^{2}\right)$ to the equation $x^{q+1}=\lambda$ for any fixed $\lambda \in G F(q)$, there are $q+1$ values of $b$ that satisfy this equation. Therefore $x$ is centralised by $q+1$ involutions sharing a common top-left entry, proving (iii).

Lemma 2.3 There are exactly $(q-2) C_{H}(t)$-orbits in $\Delta_{2}(t)$.

Proof By Lemma 2.2(i) and (ii), there are at least $(q-2) C_{H}(t)$-orbits in $\Delta_{2}(t)$. It suffices to prove that any two matrices commuting with $x$ that share a common top-left entry are $C_{H}(\langle t, x\rangle)$-conjugate. Let $g \in \Delta_{2}(t) \cap \Delta_{1}(x)$, and $a \in G F(q) \backslash\{ \pm 1\}$ be fixed such that $g_{11}=a$ and set $g_{12}=b$. By direct calculation, the diagonal entries of $g$ remain unchanged under conjugation by $C_{H}(\langle t, x\rangle)$. Let

$$
h=\left(\begin{array}{ccc}
1 & 0 & 0 \\
0 & \beta & 0 \\
0 & 0 & \beta^{-1}
\end{array}\right) \in C_{H}(\langle t, x\rangle)
$$

where $\bar{\beta} \beta=1$. Then

$$
h^{-1} g h=\left(\begin{array}{cc|c}
a & b \beta & \\
\beta^{-1} \bar{b} & -a & \\
\hline & & -1
\end{array}\right) \text {. }
$$


Clearly $b \beta$ takes $q+1$ different values for the $q+1$ different values of $\beta$. However, since there are only $q+1$ possible values for $b$, all such values are covered. That is to say, all matrices of the form

$$
\left(\begin{array}{cc|c}
a & b & \\
\bar{b} & -a & \\
\hline & -1
\end{array}\right) \in \Delta_{2}(t) \cap \Delta_{1}(x), a \neq \pm 1, \bar{b} b=1-a^{2}
$$

lie in the same $C_{H}(\langle t, x\rangle)$ orbit, and thus are all $C_{H}(t)$-conjugate. Therefore, all involutions that centralise $x$ and share a common top-left entry are $C_{H}(t)$-conjugate and so the lemma follows.

Lemma $2.4\left|\Delta_{2}(t)\right|=q\left(q^{2}-1\right)(q-2)$.

Proof Let

$$
g=\left(\begin{array}{c|cc}
-1 & & \\
& a & b \\
& \bar{b} & -a
\end{array}\right) \in \Delta_{1}(t) \text { and } h=\left(\begin{array}{cc|c}
\alpha & \beta & \\
\bar{\beta} & -\alpha & \\
\hline & & -1
\end{array}\right) \in \Delta_{2}(t) \cap \Delta_{1}(x)
$$

for $\alpha \neq \pm 1$ and $\beta \bar{\beta}=1-\alpha^{2}$ fixed. Then

$$
g h=\left(\begin{array}{ccc}
-\alpha & a \beta & b \beta \\
-\bar{\beta} & -a \alpha & -b \alpha \\
0 & -\bar{b} & a
\end{array}\right) \quad \text { and } \quad h g=\left(\begin{array}{ccc}
-\alpha & -\beta & 0 \\
a \bar{\beta} & -a \alpha & -b \\
0 & -\bar{b} \alpha & a
\end{array}\right)
$$

If $[g, h]=1$ then $a \bar{\beta}=-\bar{\beta}$ and $b \beta=0$ imply $a=-1$ and $b=0$, since $\beta \neq 0$. Therefore, $g=x$ and thus $h$ commutes with a single element of $\Delta_{1}(t)$. Since $\Delta_{1}(t)$ is a single $C_{H}(t)$ orbit, and combining Lemmas 2.1(iii) and 2.2(iii), all $C_{H}(t)$-orbits in $\Delta_{2}(t)$ have length $q(q-1)(q+1)=q\left(q^{2}-1\right)$. Hence $\left|\Delta_{2}(t)\right|=q\left(q^{2}-1\right)(q-2)$, since $\Delta_{2}(t)$ is a partition of $C_{H}(t)$-orbits.

For each $\alpha \in G F(q) \backslash\{ \pm 1\}$, define $\Delta_{2}^{\alpha}(t)$ to be the $C_{H}(t)$-orbit in $\Delta_{2}(t)$ consisting of matrices with top-left entry $\alpha \in G F(q) \backslash\{ \pm 1\}$. By Lemmas 2.1(i) and 2.2(iii), $\Delta_{2}^{\alpha}(t)$ can be written explicitly as

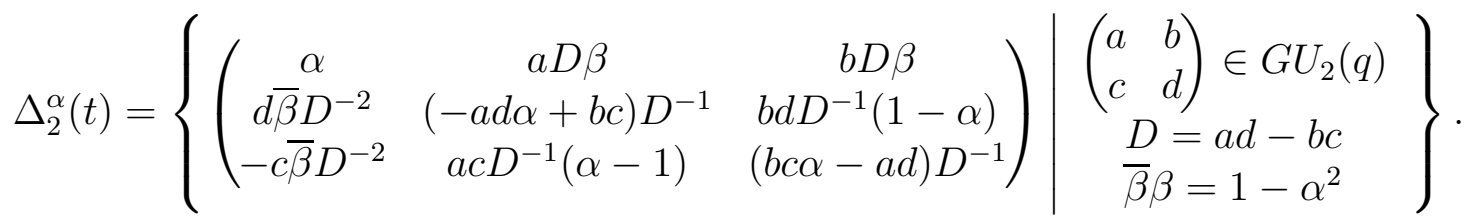




\section{Lemma 2.5 Suppose}

$$
g=\left(\begin{array}{cc|c}
\frac{\alpha}{\beta} & \beta & \\
\hline \beta & -\alpha & \\
\hline & -1
\end{array}\right) \in \Delta_{2}^{\alpha}(t) \cap \Delta_{1}(x)
$$

and

$$
h=\left(\begin{array}{ccc}
\gamma & a D \delta & b D \delta \\
d \bar{\delta} D^{-2} & (-a d \gamma+b c) D^{-1} & b d D^{-1}(1-\gamma) \\
-c \bar{\delta} D^{-2} & a c D^{-1}(\gamma-1) & (b c \gamma-a d) D^{-1}
\end{array}\right) \in \Delta_{2}^{\gamma}(t)
$$

satisfy the conditions of (2.1). If $[g, h]=1$ then

(i) $d=a \bar{\beta} \beta^{-1} \overline{\delta^{-1}} \delta D^{3}$;

(ii) if $b, c \neq 0$ then $a=-(1+\alpha)(1-\gamma)^{-1} \overline{\beta^{-1} \delta} D^{-1}$ and $b=2 D \beta^{-1}(1-\gamma)^{-1}(\beta \gamma-a \alpha \delta D) c^{-1}$; and

(iii) if $b=c=0$ then $\beta \gamma=a \alpha \delta D$.

Proof Recall that since $\alpha, \gamma \neq \pm 1$, we have $\beta, \delta \neq 0$. Direct calculation shows that

$$
g h=\left(\begin{array}{ccc}
\alpha \gamma+\beta d \bar{\delta} D^{-2} & \alpha a D \delta+\beta D^{-1}(b c-a d \gamma) & \alpha b D \delta+\beta b d D^{-1}(1-\gamma) \\
\bar{\beta} \gamma-\alpha d \bar{\delta} D^{-2} & \bar{\beta} a D \delta-\alpha D^{-1}(b c-a d \gamma) & \bar{\beta} b D \delta-\alpha b d D^{-1}(1-\gamma) \\
c \bar{\delta} D^{-2} & (1-\gamma) a c D^{-1} & -D^{-1}(b c \gamma-a d)
\end{array}\right)
$$

and

$$
h g=\left(\begin{array}{ccc}
\alpha \gamma+\bar{\beta} a D \delta & \beta \gamma-a \alpha D \delta & -b D \delta \\
\alpha d \bar{\delta} D^{-2}+\bar{\beta} D^{-1}(b c-a d \gamma) & \beta d \bar{\delta} D^{-2}-\alpha(b c-a d \gamma) D^{-1} & -b d D^{-1}(1-\gamma) \\
-\alpha c \bar{\delta} D^{-2}+\bar{\beta}(\gamma-1) a c D^{-1} & -c \beta \bar{\delta} D^{-2}-a c D^{-1} \alpha(\gamma-1) & -D^{-1}(b c \gamma-a d)
\end{array}\right) .
$$

Now if $[g, h]=1$ then we have the following relations from the $(1,1),(1,2),(1,3)$ and $(3,1)$ entries respectively:

$$
\begin{aligned}
\alpha \gamma+d \beta \bar{\delta} D^{-2} & =\alpha \gamma+\bar{\beta} a \delta D \\
a \alpha \delta D+\beta D^{-1}(b c-a d \gamma) & =\beta \gamma-a \alpha \delta D ; \\
b \alpha \delta D+b d \beta D^{-1}(1-\gamma) & =-b \delta D ; \quad \text { and } \\
-c \alpha \bar{\delta} D^{-2}+a c \bar{\beta} D^{-1}(\gamma-1) & =c \bar{\delta} D^{-2}
\end{aligned}
$$

The relations from the other entries are all equivalent to the four shown above. It is now a routine calculation to deduce parts (i)-(iii) from these relations.

Lemma 2.6 Let $y_{\alpha} \in \Delta_{2}^{\alpha}(t)$ for some $\alpha \in G F(q) \backslash\{ \pm 1\}$. Then $\left|\Delta_{1}\left(y_{\alpha}\right) \cap \Delta_{2}^{-\alpha}(t)\right|=1$. 
Proof Without loss of generality, choose $y_{\alpha}$ such that $\left[y_{\alpha}, x\right]=1$, so $\left(y_{\alpha}\right)_{11}=\alpha$ and set $\left(y_{\alpha}\right)_{12}=\beta$. Let $y_{-\alpha} \in \Delta_{2}^{-\alpha}(t)$ be as in (2.1) for suitable $a, b, c, d \in G F\left(q^{2}\right)$. We remark that if $\alpha=0$, we denote this element $y_{0}^{\prime}$ to distinguish it from $y_{0}$. Assuming $\left[y_{-\alpha}, y_{\alpha}\right]=1$, we apply Lemma 2.5 by setting $\alpha=-\gamma$, and note that $\bar{\beta} \beta=\bar{\delta} \delta$. Suppose that $b, c \neq 0$, then $a$ and $b$ are as in Lemma 2.5(ii). Since $\alpha=-\gamma$, we have $a=-D^{-1} \overline{\beta^{-1} \delta}$, giving $b=2 D \beta^{-1}(1-\gamma)^{-1}\left(\beta \gamma-\overline{\beta^{-1}} \delta \gamma\right) c^{-1}$. However, $\beta \gamma-\overline{\beta^{-1}} \delta \gamma=\beta\left(\gamma-\overline{\beta^{-1}} \beta^{-1} \bar{\delta} \delta \gamma\right)=0$ since $\overline{\beta^{-1}} \beta^{-1} \bar{\delta} \delta=1$. This yields $b=0$, contradicting our original assumption. Hence $b=c=0$, giving $a$ as in Lemma 2.5(iii) and thus $a \delta \alpha D=-\beta \alpha$. Hence either $\alpha=0$ or $a=-\beta \delta^{-1} D^{-1}$.

If $\alpha \neq 0$, then $a D=-\beta \delta^{-1}$ and $d D^{-2}=-\overline{\beta \delta^{-1}}$ showing that

$$
y_{-\alpha}=\left(\begin{array}{cc|c}
\frac{-\alpha}{\beta^{2} \delta^{-1}} & -\beta^{2} \delta^{-1} & \\
\hline & \alpha &
\end{array}\right) .
$$

If $\alpha=\gamma=0$, then both $y_{0}$ and $y_{0}^{\prime}$ commute with $x$, where $\left(y_{0}\right)_{12}=\beta$ and $\left(y_{0}^{\prime}\right)_{12}=\delta$. If $y_{0}$ and $y_{0}^{\prime}$ commute, then an easy calculation shows that $\delta= \pm \beta$. Since $y_{0} \neq y_{0}^{\prime}$, we must have $\delta=-\beta$.

Hence in both cases, $y_{\alpha}$ commutes with a single element of $\Delta_{2}^{-\alpha}(t)$.

Lemma 2.7 Let $y_{\alpha} \in \Delta_{2}^{\alpha}(t)$. Then $\left|\Delta_{1}\left(y_{\alpha}\right) \cap \Delta_{2}^{\gamma}(t)\right|=q+1$ for $\alpha \neq-\gamma$.

Proof As in Lemma 2.6, choose $y_{\alpha}$ such that $\left[y_{\alpha}, x\right]=1$ with $\left(y_{\alpha}\right)_{11}=\alpha$ and set $\left(y_{\alpha}\right)_{12}=$ $\beta$. Let $y_{\gamma} \in \Delta_{2}^{\gamma}(t)$ be as in (2.1) for suitable $a, b, c, d \in G F\left(q^{2}\right)$. For brevity we remark that if $\alpha=\gamma$, then $y_{\alpha}$ and $y_{\gamma}$ will denote different elements. Assume $\left[y_{\alpha}, y_{\gamma}\right]=1$, so the relevant relations from Lemma 2.5 hold for fixed $\alpha, \beta, \gamma, \delta$ satisfying $\alpha, \gamma \in G F(q) \backslash\{ \pm 1\}$, $\bar{\beta} \beta=1-\alpha^{2}$ and $\bar{\delta} \delta=1-\gamma^{2}$.

Suppose $b=c=0$, so Lemma 2.5(iii) holds. Since $\beta \neq 0$ and if $\alpha=0$, then $\gamma=0$, contradicting the assumption that $\alpha \neq-\gamma$. Hence $a=\beta \gamma \alpha^{-1} \delta^{-1} D^{-1}$. Using Lemma 2.5(i), we get $d=\overline{\beta \delta^{-1}} D^{2} \gamma \alpha^{-1}$ and so $a d=\bar{\beta} \beta \overline{\delta^{-1}} \delta^{-1} \gamma^{2} \alpha^{-2} D$. Combining the expressions for $\bar{\beta} \beta, \bar{\delta} \delta$ and $D$, we get

$$
\left(\gamma^{2}-\alpha^{2} \gamma^{2}\right)\left(\alpha^{2}-\alpha^{2} \gamma^{2}\right)^{-1}=1,
$$

giving $\gamma^{2}=\alpha^{2}$ resulting in $\gamma= \pm \alpha$. Since $\alpha \neq-\gamma$, we must have $\alpha=\gamma$. But then $a D \delta=\beta$ and so $y_{\gamma}=y_{\alpha}$. Therefore, we may assume $b, c \neq 0$.

By a long but routine check, substitutions of $\bar{\beta} \beta, \bar{\gamma} \gamma$ and the relations in Lemma 2.5 show that $a d-b c=D$ holds. These relations also clearly show that $a, b, c$ and $d$ are all non-zero. Hence by Lemma 2.1(i), we have $\bar{a} b=-\bar{c} d$ and so $\bar{c} c=-\bar{a} b c d^{-1}$, and there are $q+1$ values of $c$ that satisfy this equation.

It now suffices to check that the remaining conditions of Lemma 2.1(i) hold. Since $\alpha, \gamma \in G F(q)$, we have $\overline{(1-\alpha)}(1-\alpha)^{-1}=\overline{(1-\gamma)}(1-\gamma)^{-1}=1$. Together with the relations already determined, we have $\bar{a} a+\bar{c} c=\bar{a} a-\bar{a} d^{-1} b c=\overline{D^{-1}} D^{-1}$. However 
$\bar{D} D=1$, so the conditions of Lemma 2.1(i) hold. By considering $\overline{\bar{a} a+\bar{c} c}$, we get a similar result for $\bar{b} b+\bar{d} d$. Hence there is only one possible value of each of $a$ and $d$, there are $(q+1)$ different values of $c$ with $b$ depending on $c$, proving the lemma.

As a consequence, we have the following.

Corollary 2.8 Let $y \in \Delta_{2}(t)$. Then $\left|\Delta_{1}(y) \cap \Delta_{3}(t)\right|=q+1$.

Proof Since the valency of the graph is $q(q-1)$ and $\left|\Delta_{1}(y) \cap \Delta_{1}(t)\right|=1$, Lemmas 2.6 and 2.7 give Corollary 2.8 .

For the remainder of this paper, denote

$$
y=\left(\begin{array}{rrr}
0 & 1 & 0 \\
1 & 0 & 0 \\
0 & 0 & -1
\end{array}\right) \in \Delta_{2}^{0}(t)
$$

and define

$$
z_{\gamma}=\left(\begin{array}{rrr}
1 & -2 & \bar{\gamma} \\
-2 & 1 & -\bar{\gamma} \\
\gamma & -\gamma & -3
\end{array}\right),
$$

for $\bar{\gamma} \gamma=-4$. An easy check shows that $\left[z_{\gamma}, y\right]=1,{\overline{z_{\gamma}}}^{T}=z_{\gamma}$ and $z_{\gamma}$ is an involution, hence $z_{\gamma} \in X$ and $d\left(t, z_{\gamma}\right) \leq 3$. However, since $t$ is the sole element with top-left entry 1 that is at most distance 2 from $t$, we have $d\left(t, z_{\gamma}\right) \geq 3$ and thus equality.

Lemma $2.9 \Delta_{1}(y) \cap \Delta_{3}(t)=\left\{z_{\gamma} \mid \gamma \in G F\left(q^{2}\right), \bar{\gamma} \gamma+4=0\right\}$.

Proof There are $q+1$ values of $\gamma$ and $z_{\gamma}$ centralises $y$ for all such $\gamma$. By Corollary 2.8, $\left|\Delta_{1}(y) \cap \Delta_{3}(t)\right|=q+1$, and so the lemma follows.

Fix $\gamma$ and let $g \in C_{H}(t)$ be of the form as described in Lemma 2.1(i) for suitable $a, b, c, d \in G F\left(q^{2}\right)$. Then

$$
z_{\gamma} g=\left(\begin{array}{ccc}
D^{-1} & -2 a+c \bar{\gamma} & -2 b+d \bar{\gamma} \\
-2 D^{-1} & a-\bar{\gamma} c & b-d \bar{\gamma} \\
\gamma D^{-1} & -\gamma a-3 c & -b \gamma-3 d
\end{array}\right)
$$

and

$$
g z_{\gamma}=\left(\begin{array}{ccc}
D^{-1} & -2 D^{-1} & D^{-1} \bar{\gamma} \\
-2 a+b \gamma & a-b \gamma & -a \bar{\gamma}-3 b \\
-2 c+d \gamma & c-d \gamma & -c \bar{\gamma}-3 d
\end{array}\right) .
$$

If $\left[z_{\gamma}, g\right]=1$, then we equate the entries to get conditional relations. From the $(2,2)$ entries, we see that $b=c \bar{\gamma} \gamma^{-1}$. This, combined with the $(2,3)$ entry, gives $d=a+4 c \gamma^{-1}$. 
The $(3,1)$ entry shows that $c=-2^{-1}\left(D^{-1}-d\right) \gamma$, and so $d=2 D^{-1}-a$. Hence

$$
\begin{aligned}
& b=-2^{-1}\left(a-D^{-1}\right) \bar{\gamma} ; \\
& c=-2^{-1}\left(a-D^{-1}\right) \gamma ; \quad \text { and } \\
& d=2 D^{-1}-a
\end{aligned}
$$

for $a \in G F\left(q^{2}\right)$. A routine check shows these relations are sufficient for $\left[z_{\gamma}, g\right]=1$. These relations, together with the conditions of Lemma 2.1(i) and $\bar{D} D=1$, give

$$
a \overline{D^{-1}}+\bar{a} D^{-1}=2 .
$$

Clearly, the number of possible such $a$ is $\left|C_{H}\left(\left\langle t, z_{\gamma}\right\rangle\right)\right|$. Since $D=a d-b c$, we get $D^{3}=1$. Therefore $\bar{D} D=D^{3}=1$ which has a solution $D \neq 1$ if and only if $q \equiv 5(\bmod 6)$.

Lemma 2.10 If $q \not \equiv 5(\bmod 6)$, then $\left|C_{H}\left(\left\langle t, z_{\gamma}\right\rangle\right)\right|=q$. Moreover, $\mathcal{C}(H, X)$ is connected of diameter 3 and $\left|\Delta_{3}(t)\right|=(q+1)\left(q^{2}-1\right)$.

Proof Since $q \not \equiv 5(\bmod 6)$, from $(2.2)$ we have $D=1$ and $\bar{a}+a-2=0$. There are $q$ distinct values of $a$ satisfying this, so $\left|C_{H}\left(\left\langle t, z_{\gamma}\right\rangle\right)\right|=q$. Denote the $C_{H}(t)$-orbit containing $z_{\gamma}$ by $\Delta_{3}^{\gamma}(t)$. Hence,

$$
\left|\Delta_{3}^{\gamma}(t)\right|=\frac{\left|C_{H}(t)\right|}{\left|C_{H}\left(\left\langle t, z_{\gamma}\right\rangle\right)\right|}=(q+1)\left(q^{2}-1\right) .
$$

Combining Lemmas 2.1(ii)-(iii) and 2.4, we have

$$
\left|X \backslash\left(\{t\} \cup \Delta_{1}(t) \cup \Delta_{2}(t)\right)\right|=\left|\Delta_{3}^{\gamma}(t)\right| .
$$

Hence $\mathcal{C}(H, X)$ is connected of diameter 3 , and $\Delta_{3}^{\gamma}(t)=\Delta_{3}(t)$ as required.

Remark Since $\Delta_{3}(t)$ is a single $C_{H}(t)$-orbit and the valency of the graph is $q(q-1)$, for $w \in \Delta_{3}(t)$ we have $\left|\Delta_{1}(w) \cap \Delta_{3}(t)\right|=q$. This proves Theorem 1.1 when $q \not \equiv 5(\bmod 6)$.

We now turn our attention to the remaining case, when $q \equiv 5(\bmod 6)$.

Lemma 2.11 Suppose $q \equiv 5(\bmod 6)$.

(i) $\left|C_{H}\left(\left\langle t, z_{\gamma}\right\rangle\right)\right|=3 q$.

(ii) There are exactly three $C_{H}(t)$-orbits in $\Delta_{3}(t)$, each of length $\frac{1}{3}(q+1)\left(q^{2}-1\right)$.

(iii) $\mathcal{C}(H, X)$ is connected of diameter 3 and $\left|\Delta_{3}(t)\right|=(q+1)\left(q^{2}-1\right)$. 
Proof From (2.2), we have $\bar{D} D=D^{3}=1$ and since $q \equiv 5(\bmod 6)$, there are three possible values for $D$. Since $a \overline{D^{-1}}+\bar{a} D^{-1}-2=\overline{\left(a \overline{D^{-1}}\right)}+a \overline{D^{-1}}-2=0$ then for each value of $D$, there are $q$ such values of $a \overline{D^{-1}}$. Hence there are $3 q$ values of $a \overline{D^{-1}}$ in total, proving (i).

Fix $\gamma$, and let $\Delta_{3}^{\gamma}(t)$ be the $C_{H}(t)$-orbit containing $z_{\gamma}$. We have

$$
\left|\Delta_{3}^{\gamma}(t)\right|=\frac{\left|C_{H}(t)\right|}{\left|C_{H}\left(\left\langle t, z_{\gamma}\right\rangle\right)\right|}=\frac{1}{3}(q+1)\left(q^{2}-1\right)
$$

Let $h=\left(\begin{array}{l|ll}E & & \\ & \lambda & \mu \\ \sigma & \tau\end{array}\right) \in C_{H}(t)$ where $E=\lambda \tau-\mu \sigma$. Then

$h^{-1} z_{\gamma} h=\left(\begin{array}{ccc}1 & E(\bar{\gamma} \sigma-2 \lambda) & E(-2 \mu+\tau \bar{\gamma}) \\ -E^{-2}(2 \tau+\mu \gamma) & (\lambda \mu \gamma-\sigma \bar{\gamma} \tau+4 \mu \sigma) E^{-1}+1 & \left(-\bar{\gamma} \tau^{2}+\mu^{2} \gamma+4 \mu \tau\right) E^{-1} \\ E^{-2}(2 \sigma+\lambda \gamma) & \left(-\lambda^{2} \gamma+\sigma^{2} \bar{\gamma}-4 \lambda \sigma\right) E^{-1} & (\lambda \mu \gamma-\sigma \bar{\gamma} \tau+4 \mu \sigma) E^{-1}-3\end{array}\right)$.

Suppose $h^{-1} z_{\gamma} h=z_{\delta} \in \Delta_{3}(t) \cap \Delta_{1}(y)$ for some $\delta \neq \gamma$. Hence $\left(h^{-1} z_{\gamma} h\right)_{21}=-2=$ $\left(h^{-1} z_{\gamma} h\right)_{12}$ gives $\tau=E^{2}-2^{-1} \mu \gamma$ and $\lambda=2^{-1} \bar{\gamma} \sigma+E^{-1}$. Since $E=\lambda \tau-\mu \sigma$, we have $2^{-1} \bar{\gamma} \sigma E^{2}-2^{-1} \mu \gamma E^{-1}=0$ and so $\mu=\bar{\gamma} \gamma^{-1} \sigma E^{3}$. Rewriting $\tau$, we get $\tau=E^{2}-2^{-1} \bar{\gamma} \sigma E^{3}$. To summarise,

$$
\begin{aligned}
& \lambda=2^{-1} \bar{\gamma} \sigma+E^{-1} ; \\
& \mu=\bar{\gamma} \gamma^{-1} \sigma E^{3} ; \quad \text { and } \\
& \tau=E^{2}-2^{-1} \bar{\gamma} \sigma E^{3} .
\end{aligned}
$$

Using these relations and $\bar{\gamma} \gamma=-4$, a simple check shows that $\left(h^{-1} z_{\gamma} h\right)_{22}=1$ and $\left(h^{-1} z_{\gamma} h\right)_{33}=-3$ hold, and $\left(h^{-1} z_{\gamma} h\right)_{31}=E^{-3} \gamma=\delta$. Easy substitutions and checks show that $\left(h^{-1} z_{\gamma} h\right)_{32}=-\left(h^{-1} z_{\gamma} h\right)_{31}$ and $\overline{\left(h^{-1} z_{\gamma} h\right)_{13}}=\left(h^{-1} z_{\gamma} h\right)_{31}$. Since $\bar{\delta} \delta=-4$, we have $\overline{E^{3}} E^{3}=1$. In particular, $E^{3}$ is a $(q+1)^{\text {th }}$ root of unity. There are $q+1$ such roots and only a third of them are cubes in $G F\left(q^{2}\right)^{*}$. Hence there are only $\frac{1}{3}(q+1)$ such values of $\delta=E^{-3} \gamma$. Therefore, we can pick $\gamma_{1}, \gamma_{2}$ and $\gamma_{3}$ such that $\overline{\gamma_{i}} \gamma_{i}=-4$ where the $z_{\gamma_{i}}$ are not pairwise $C_{H}(t)$-conjugate. Hence there are at least 3 orbits in $\Delta_{3}(t)$, and by $(2.3)$ they all have length $\frac{1}{3}(q+1)\left(q^{2}-1\right)$. But (as in the proof of Lemma 2.10), $\left|X \backslash\left(\{t\} \cup \Delta_{1}(t) \cup \Delta_{2}(t)\right)\right|=(q+1)\left(q^{2}-1\right)$ and so this proves (ii), and (iii) follows immediately.

This now completes the proof of Theorem 1.1. 


\section{References}

[1] Aschbacher, M. A condition for the existence of a strongly embedded subgroup. Proc. Amer. Math. Soc. 38 (1973), 509-511.

[2] Bates, C.; Bundy, D.; Perkins, S.; Rowley, P. Commuting involution graphs for symmetric groups. J. Algebra 266 (2003), no. 1, 133-153.

[3] Bates, C.; Bundy, D.; Perkins, S.; Rowley, P. Commuting involution graphs for finite Coxeter groups. J. Group Theory 6 (2003), no. 4, 461-476.

[4] Bates, C.; Bundy, D.; Perkins, S.; Rowley, P. Commuting involution graphs in special linear groups. Comm. Algebra 32 (2004), no. 11, 4179-4196.

[5] Bates, C.; Bundy, D.; Hart, S.; Rowley, P. Commuting involution graphs for sporadic simple groups. J. Algebra 316 (2007), no. 2, 849-868.

[6] Brauer, R.; Fowler, K. A. On groups of even order. Ann. of Math. (2) 62 (1955), $565-583$.

[7] Everett, A. Commuting Involution Graphs of Certain Finite Classical Groups. PhD Thesis, University of Manchester, 2010.

[8] Everett, A.; Rowley, P. Commuting Involution Graphs for 4-dimensional Projective Symplectic Groups. http://eprints.ma.man.ac.uk/1564/; (preprint).

[9] Fischer, B. Finite groups generated by 3-transpositions. University of Warwick Lecture Notes, 1969.

[10] Gorenstein, D. Finite groups. Second edition. Chelsea Publishing Co., New York, 1980.

[11] Rapinchuk, A.S.; Segev, Y.; Seitz, G.M. Finite quotients of the multiplicative group of a finite dimensional division algebra are solvable. J. Amer. Math. Soc. 15 (2002), no. 4, 929-978 (electronic).

[12] Taylor, P. Computational Investigation into Finite Groups. PhD Thesis, University of Manchester, 2010.

[13] Wall, G. E. On the conjugacy classes in the unitary, symplectic and orthogonal groups. J. Austral. Math. Soc. 3 (1963), 1-62.

[14] Wright, B. Graphs Associated with the sporadic simple groups $F i_{24}^{\prime}$ and $B M$. PhD Thesis, University of Manchester, 2010. 